\title{
Braiding with magnetic octupoles
}

\author{
Thomas Lachner $\odot,{ }^{1, *}$ Daniel de las Heras $\odot,{ }^{2}$ and Thomas M. Fischer $\odot^{1}$ \\ ${ }^{1}$ Experimentalphysik X, Physikalisches Institut, Universität Bayreuth, D-95440 Bayreuth, Germany \\ ${ }^{2}$ Theoretische Physik II, Physikalisches Institut, Universität Bayreuth, D-95440 Bayreuth, Germany
}

(Received 22 June 2020; accepted 8 December 2020; published 13 January 2021)

\begin{abstract}
We simulate the trajectories of magnetic octupole colloids driven by periodic loops of an external magnetic field and placed above a two-dimensional threefold symmetric magnetic pattern. The octupoles avoid the threefold symmetric points above the lattice, either in a topologically trivial way, or by nontrivially winding around these high symmetry points both with respect to their position and with respect to their orientation. We calculate the full dynamical phase space of this winding behavior by changing both lattice symmetries and modulation loops. We further use the nontrivial topology to braid with octupoles and we supply a protocol for both braiding and weaving with such microscopic particles. Our classical external field command should work equally well for quantum mechanical octupoles on magnetic nanopatterns, providing an explicit protocol for the exchange of anyonic quantum particles.
\end{abstract}

DOI: 10.1103/PhysRevResearch.3.013043

\section{INTRODUCTION}

Robust and precise transport of colloidal particles enables their use for a variety of applications such as, e.g., lab-ona-chip devices [1] and drug delivery with colloidal carriers [2]. Colloidal transport can be achieved via the application of global external fields $[3,4]$ such as gravitational, electric, and magnetic fields. As a result a collection of colloidal particles is, in general, simultaneously transported along the same direction. If the colloidal particles are subject to a periodic, time-dependent, external potential the transport can be of topological nature. That is the case of magnetic colloidal particles above a periodic magnetic pattern and driven by a uniform time-dependent magnetic field [5-8]. There, the orientation of the uniform external field varies periodically in time performing closed loops. There exist special loops such that once the field returns to the initial orientation, the colloids have been transported by one unit cell of the pattern. The transport is robust against perturbations since it depends only on global topological invariants. Moreover, despite a single magnetic external field acts on all particles, the complex topology of the potential landscape allows the simultaneous and independent transport of colloidal particles in different directions, provided that the particles differ in either their shape [9] or their magnetic susceptibilities [10]. In Ref. [9] we provide a recipe for individually transporting paramagnetic bipeds (rods of different length assembled from a collection of paramagnetic colloids) into different directions. In

\footnotetext{
*Thomas.Lachner@uni-bayreuth.de

Published by the American Physical Society under the terms of the Creative Commons Attribution 4.0 International license. Further distribution of this work must maintain attribution to the author(s) and the published article's title, journal citation, and DOI.
}

Ref. [10] we simultaneously transported paramagnetic particles, diamagnetic particles, and induced quadrupolar particles (consisting of a paramagnetic particle bound to a diamagnetic particle) in different directions [10]. Simultaneous and independent transport along different directions is possible because different particles fell into different topological classes.

Here, we go a fundamental step further and present a system where simultaneous and independent motion of identical colloidal particles is achieved with a single magnetic field. The particles are induced colloidal octupoles located above a periodic magnetic pattern. The highly nontrivial topology of the octupole potential created by the magnetic pattern and the external uniform field allows the independent and robust motion control of identical particles. We use this feature to create braids by consecutively exchanging two octupoles in a topologically protected way.

A mathematical braid results from the consecutive permutations of two neighboring strings $i$ and $i+1$, where the $i$ th string crosses the neighboring $(i+1)$ th string on the top (bottom) of the other. The sequence of positive (top) and negative (bottom) permutations characterizes the topology of the braid when considering both ends of all strings fixed. An example of a mathematical braid is shown in Fig. 1(a). There are different sequences of permutations having the same topology and it is thus possible to deform one sequence into an equivalent sequence without cutting any of the strings. Braids play an important role in robust quantum computation [11] with two-dimensional quasiparticles [12] and in the description of complex fluid dynamical problems $[13,14]$. Braids can characterize structural properties [15] such as tertiary structures of polymers [16-18], defect lines in nematic liquid crystals $[19,20]$, and optical vortices [21], but also dynamic classical trajectories of particles [22], solitons [23], magnetic holes [24], and animals in a flock [25]. Braids can also be formed using active colloids [26]. 


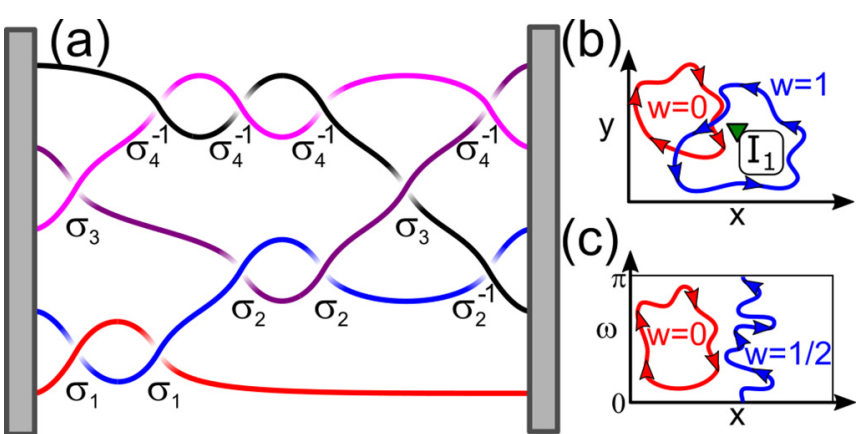

FIG. 1. (a) A mathematical braid consists of permutation of strings. There are five strings. The permutation $\sigma_{1}$ moves the string at position 1 to the position 2 above the string that is moved in the opposite direction. The permutation $\sigma_{4}^{-1}$ moves the string at position 4 to the position 5 below the string that is moved in the opposite direction. (b) A trajectory of winding number $w=0$ and $w=1$ around a point $I_{1}$ in translational space. (c) A closed trajectory of orientational winding number $w=0$ and $w=1 / 2$ in the space spanned by an orientational angle $\omega$ and a spatial coordinate $x$. For an object with two perpendicular mirror planes, such as a liquid crystal or an octupole, the orientation angle $\omega$ is $\pi$ periodic.

Braids are an example of a topologically nontrivial state. Topology is also used to describe complex states in other areas of physics such as, e.g., in quantum electronics. There, topological states arise for Bloch electrons that are driven adiabatically through the Brillouin zone. These topological states are characterized by topological invariants called Chern numbers (a higher dimensional generalization of winding numbers) or for the case of Floquet systems by winding numbers. The classical analog of such quantum system is a system driven periodically in some external parameter space (here called the control space $\mathcal{C}$ ). The corresponding topological invariants are winding numbers of the intrinsic variables of the system that count how often a closed trajectory in control space winds around a hole. Examples of winding numbers are shown in Figs. 1(b) and 1(c). We distinguish winding numbers of translational degrees of freedom and winding numbers of rotational degrees of freedom. The latter winding numbers are commonly used to, e.g., describe the topological charge of defects of the director field in liquid crystals [27].

The paper is organized as follows. In Sec. II we introduce the model for the colloidal octupoles and describe the magnetostatic potential (in the point octupole approximation) created by the periodic magnetic pattern. We introduce a family of threefold symmetric magnetic patterns. Each pattern is characterized by a phase and described in terms of a magnetostatic potential. Section III is dedicated to the results. We discuss the mathematical, topological, and symmetry aspects of both the magnetic field created by the pattern and the octupole potential. We provide a dynamical phase diagram of the driven motion of octupoles and show how to enable individual and simultaneous control of identical particles. We end the Results section providing an explicit modulation loop that accomplishes the exchange of the positions of two identical octupoles. As examples of simultaneous control of identical octupoles, we simulate braiding and weaving trajectories

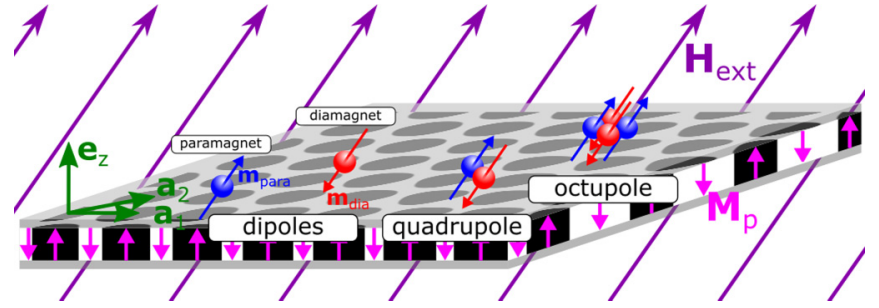

FIG. 2. Scheme of a magnetic pattern with magnetization $\mathbf{M}_{\mathrm{p}}$ exhibiting a sixfold point symmetry. Paramagnetic (blue) and diamagnetic (red) colloids are placed above the pattern (restricted to move on a plane parallel to the pattern). Individual colloids responding as induced dipoles, bound pairs responding as induced quadrupoles, and a bound quadruple responding as an induced octupole to the external magnetic field are represented.

using three and four octupoles, respectively. We conclude in Sec. IV.

\section{MODEL}

We use the modulation of the orientation of a homogeneous time-dependent external magnetic field $\mathbf{H}_{\text {ext }}(t)$ of constant magnitude to drive the motion of colloidal octupoles above a threefold symmetric pattern (schematics are shown in Figs. 2 and in 3). The set of all possible orientations of $\mathbf{H}_{\text {ext }}$ defines the surface of a sphere that we call the control space $\mathcal{C}$. The octupoles are confined to a plane parallel and above the threefold symmetric magnetic pattern. The particles respond to the adiabatic changes of $\mathbf{H}_{\text {ext }}$ by changing both their position and their orientation. We call action space $\mathcal{A}$ to the plane where the octupoles lie. We show here how a winding in control space causes topologically nontrivial winding of the particles in action space.

A magnetic dipole moment $\mathbf{m}_{\text {para }}\left(\mathbf{m}_{\text {dia }}\right)$ parallel (antiparallel) to the magnetic field $\mathbf{H}$ is induced in a single paramagnetic (diamagnetic) colloid by the total magnetic field $\mathbf{H}=\mathbf{H}_{\mathrm{ext}}(t)+\mathbf{H}_{\mathrm{p}}\left(\mathbf{r}_{\mathcal{A}}\right)$, as schematically represented in Fig. 2. Here $\mathbf{H}_{\mathrm{p}}\left(\mathbf{r}_{\mathcal{A}}\right)$ is the time-independent magnetic field created by the magnetic pattern. The magnitude of the pattern field is much smaller than that of the external field, $H_{\mathrm{p}} \ll H_{\text {ext }}$, and hence the induced dipolar moment is almost parallel (antiparallel) to the external field. A bound pair of a paramagnetic and a diamagnetic colloid of exactly opposite susceptibilities, connected in the direction of the unit vector $\mathbf{e}_{\mathrm{pd}}$, forms an induced quadrupole (see Fig. 2), while four colloids arranged on a square with paramagnets and diamagnets on the opposite diagonals of the square (as shown in Fig. 2) form an induced octupole. Such octupoles created from paramagnetic and diamagnetic colloids [28] with exactly the opposite susceptibility (such that the dipole moment vanishes) ordered in an improper fourfold symmetric arrangement [29] (further killing the quadrupolar moment) leaves only an octupole moment $q_{\text {oct }} \mathbf{H}_{\mathrm{ext}}\left(\mathbf{e}_{\mathrm{p}} \mathbf{e}_{\mathrm{p}}-\mathbf{e}_{\mathrm{d}} \mathbf{e}_{\mathrm{d}}\right)$. Here $\mathbf{e}_{\mathrm{p}}$ and $\mathbf{e}_{\mathrm{d}}$ are the unit vectors of the paramagnetic and diamagnetic diagonals of the square, as shown in Fig. 3. Experimentally, induced colloidal octupoles can be built from paramagnetic and diamagnetic colloidal particles generalizing the scheme used in Ref. [10] for manufacturing induced quadrupolar colloidal particles. 


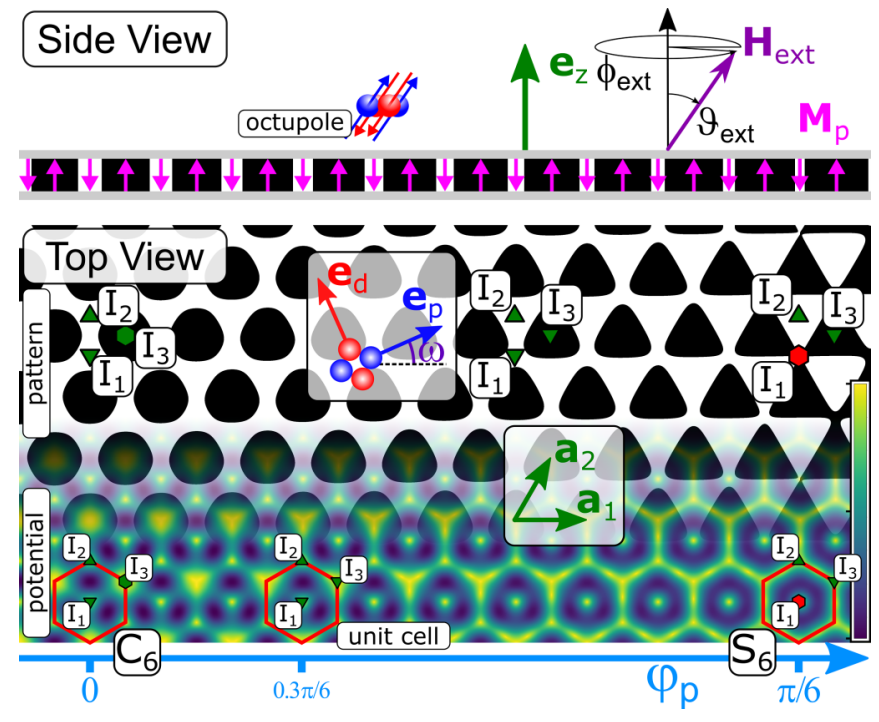

FIG. 3. Side view: Scheme of an octupole built from paramagnetic (blue) and diamagnetic (red) colloidal particles with induced dipole moments (blue and red arrows) above a magnetic pattern with magnetization $\mathbf{M}_{\mathrm{p}}$ in an external field $\mathbf{H}_{\mathrm{ext}}$ (characterized by $\phi_{\text {ext }}, \vartheta_{\text {ext }}$ ). Top view: Same octupole with paramagnetic (diamagnetic) axes $\mathbf{e}_{\mathrm{p}}\left(\mathbf{e}_{\mathrm{d}}\right)$ and orientation $\omega$ above the threefold symmetric magnetic pattern changing with pattern phase $\varphi_{\mathrm{p}}$. The color-coded octupole potential (blue $=$ minima, yellow $=$ maxima) is plotted in arbitrary units for an external field $\mathbf{H}_{\mathrm{ext}}$ pointing along the $\mathbf{e}_{z}$ direction. The threefold symmetric points $I_{1}, I_{2}$, and $I_{3}$ of the pattern (green triangles) are maxima of the potential and hence are avoided by the octupoles. At certain pattern symmetries one of these points acquires a proper (green hexagon) or improper (red hexagon) sixfold symmetry. $\mathbf{a}_{1}$ and $\mathbf{a}_{2}$ are the primitive lattice vectors. Illustrative unit cells are framed red.

The octupole is confined to a plane $z=$ const parallel to the magnetic pattern and oriented such that $\mathbf{e}_{\mathrm{p}} \times \mathbf{e}_{\mathrm{d}}=\mathbf{e}_{z}$, i.e., the third axis of the octupole points along the normal to the pattern (see Fig. 3). Such arrangement can be achieved experimentally via, e.g., sedimentation [5] or magnetic boundary effects [28]. The lateral position $\mathbf{r}_{\mathcal{A}}$ of the octupole and its orientation angle $\omega=\angle\left(\mathbf{e}_{\mathrm{p}}, \mathbf{a}_{1}\right)$ between the paramagnetic axis and the lattice primitive vector $\mathbf{a}_{1}$ are allowed to arrange as to minimize the potential $U_{\text {oct }}$ generated by both the external field and the pattern. We approximate $U_{\text {oct }}$ by the potential of an induced point octupole:

$$
U_{\mathrm{oct}}=-q_{\mathrm{oct}} H_{\mathrm{ext}}^{\alpha}\left(e_{\mathrm{p}}^{\beta} e_{\mathrm{p}}^{\gamma}-e_{\mathrm{d}}^{\beta} e_{\mathrm{d}}^{\gamma}\right) \partial_{\alpha} \partial_{\beta} \partial_{\gamma} \psi_{\mathrm{p}},
$$

which is the third term in a multipole expansion of an arbitrary arrangement of colloidal particles. Note that in general the multipole expansion also involves a dipolar term $U_{\text {dip }}=-q_{\text {dip }} H_{\text {ext }}^{\alpha} \partial_{\alpha} \psi_{\mathrm{p}}$ and a quadrupolar term $U_{\text {quad }}=$ $-q_{\text {quad }} H_{\mathrm{ext}}^{\alpha} e_{\mathrm{pd}}^{\beta} \partial_{\alpha} \partial_{\beta} \psi_{\mathrm{p}}$. However, both terms vanish here since $q_{\text {dip }}=q_{\text {quad }}=0$ for an octupole like that in Fig. 2. In Eq. (1), $q_{\text {oct }} H_{\text {ext }}$ is the modulus of the induced magnetic octupole moment $q_{\text {oct }} \mathbf{H}_{\text {ext }}\left(\mathbf{e}_{\mathrm{p}} \mathbf{e}_{\mathrm{p}}-\mathbf{e}_{\mathrm{d}} \mathbf{e}_{\mathrm{d}}\right)$, there is a summation over repeated Cartesian indices $\{\alpha, \beta, \gamma\}$, and $\psi_{\mathrm{p}}$ is the magnetostatic potential of the magnetic pattern given by

$$
\psi_{\mathrm{p}} \propto-e^{-Q z} \sum_{n=0}^{2} \cos \left(\left[\mathcal{R}_{3}^{n} \cdot \mathbf{Q}\right] \cdot \mathbf{r}_{\mathcal{A}}-\varphi_{\mathrm{p}}\right) .
$$

Here $\mathcal{R}_{3}$ denotes a rotation by $2 \pi / 3$ around the $z$ axis, and $\mathbf{Q}$ is one of the primitive reciprocal lattice vectors of the magnetic pattern. We refer the reader to Ref. [5] for further details about the magnetostatic potential. We remark here that the approximations that enter in Eqs. (1) and (2) are accurate provided that $H_{\mathrm{p}} \ll H_{\mathrm{ext}}$ and that the particles are sufficiently far from the pattern (a distance comparable to or larger than the size of the unit cell). The magnetic field of the magnetic pattern is given by $\mathbf{H}_{\mathrm{p}}=\nabla \psi_{\mathrm{p}}$.

The pattern phase $\varphi_{\mathrm{p}}$ causes an entire family of threefold magnetic patterns. In Fig. 3 we show the entire family of patterns by slowly increasing $\varphi_{\mathrm{p}}$ from $\varphi_{\mathrm{p}}=0$ toward $\varphi_{\mathrm{p}}=\pi / 6$. There are three points $I_{1}, I_{2}$, and $I_{3}$ with threefold symmetry in each unit cell. For particular values of $\varphi_{\mathrm{p}}$ one of these points acquires a higher proper (green hexagon in Fig. 3) or improper (red hexagon in Fig. 3) sixfold symmetry. The magnetic patterns can be experimentally created using, e.g., lithographic patterning of thin magnetic films [30,31]. In Ref. [5] we have used such patterns with typical lattice constants of $a \approx 7 \mu \mathrm{m}$ to experimentally study the transport of both diamagnetic and paramagnetic particles.

The orientation of the uniform external magnetic field $\mathbf{H}_{\mathrm{ext}}$ $\left(H_{\text {ext }} \gg H_{\mathrm{p}}\right)$ is characterized by the tilt angle $\vartheta_{\text {ext }}$ with respect to the pattern normal and by the azimuth angle $\phi_{\text {ext }}$.

\section{RESULTS}

We use overdamped Brownian dynamics simulations to characterize the motion of the colloidal octupoles. The modulated external field $\mathbf{H}_{\mathrm{ext}}(t)$ is given as a closed loop. The period of one closed loop of the external field is much larger than the characteristic relaxation time of the octupole. Therefore, the particles can follow the potential adiabatically, which is possible except if a ratchetlike event occurs. Further details about the Brownian dynamics simulations can be found in the Appendix.

\section{A. Dynamical phase diagram}

We are interested in both the position and the orientation $\left(\mathbf{r}_{\mathcal{A}}, \omega\right)$ of the octupole as we adiabatically vary the orientation of the external magnetic field along a closed loop. For any phase pattern, the periodic octupole potential, Eq. (1), has always at least one minimum per unit cell that cannot disappear (independently of the orientation of the external field). Such minimum usually moves in a continuous way as we vary the orientation of the external magnetic field. The result is an adiabatic motion of the octupole that follows the trajectory of the potential minimum. However, if the number of minima per unit cell differs from one, then the additional, excess minima, can disappear at points of instability that might occur at specific orientations of the external field. If an excess minimum is occupied by an octupole at the instability, then the octupole can no longer move adiabatically but jumps into a remaining minimum at a different location. We thus distinguish adiabatic trajectories and ratchet trajectories that 


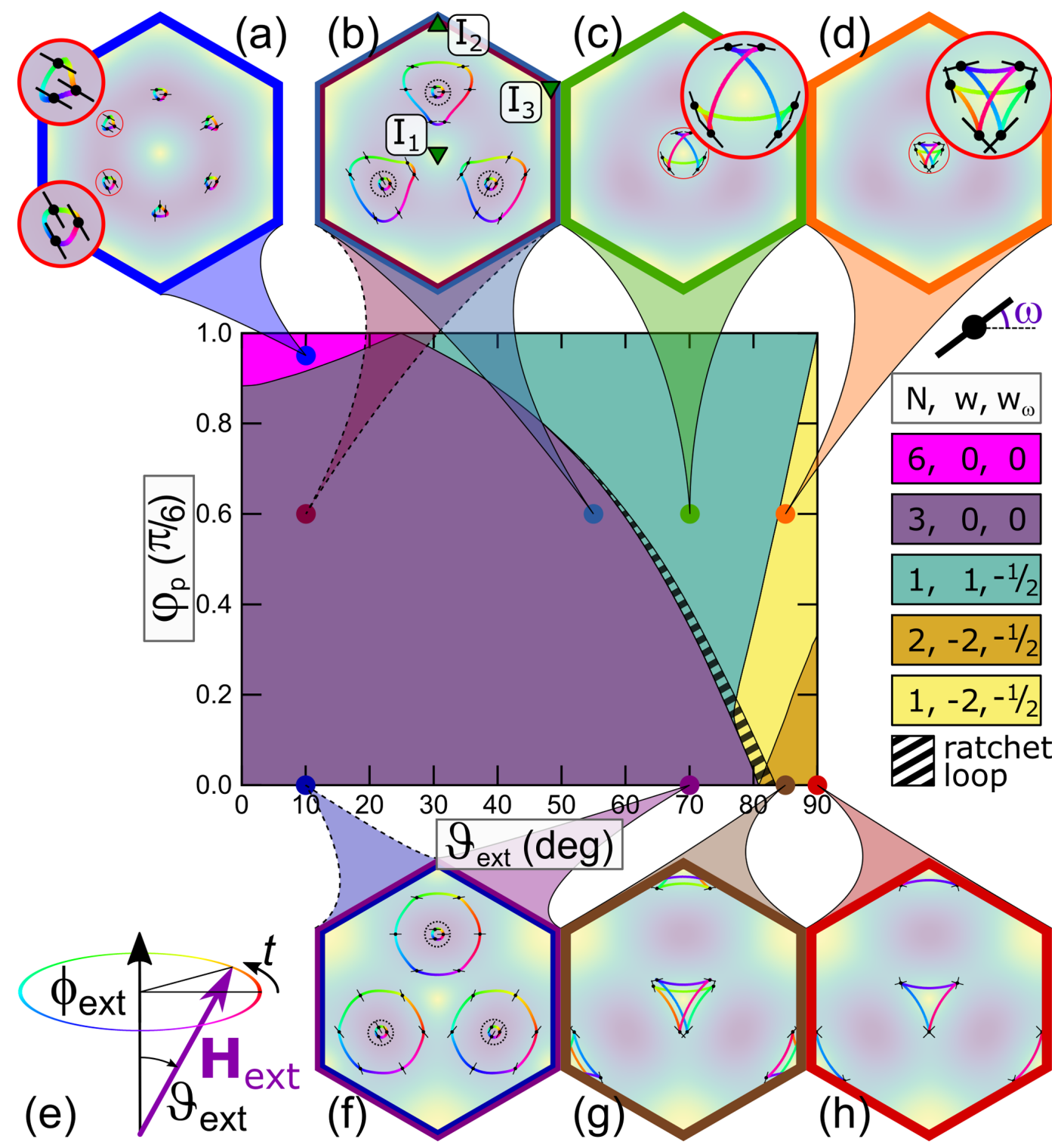

FIG. 4. Dynamic phase diagram in the plane of precession angle $\vartheta_{\text {ext }}$ and pattern phase $\varphi_{\mathrm{p}}$. Each dynamical phase is characterized by the number of stable coexisting loops $N$ together with the winding number $w=w_{1}+w_{2}+w_{3}$ around the threefold symmetric points of the lattice, and the orientational winding number $w_{\omega}$. Most phases are adiabatic (solid colors), where the octupole is always quasistable. The ratchet phases (textured) contain trajectories where a potential minimum converts to a saddle point and the octupole jumps into a new stable position with a speed independent of the speed of the external field. The panels (a)-(d) and (f)-(g) show coexisting trajectories in a unit cell. In panels (b) and (f) we show trajectories corresponding to two different values of $\vartheta_{\text {ext }}$ as indicated by the two different points in the same phase (the smaller trajectories correspond to the point at smaller $\vartheta_{\text {ext }}$ ). Panels (a), (c), and (d) have insets with magnified trajectories. The location of the threefold symmetric points $I_{1}, I_{2}$, and $I_{3}$ can be found in panel (b). Panel (e) shows a driving external field loop together with the definition of the angles $\vartheta_{\text {ext }}$ and $\phi_{\text {ext }}$ characterizing the loop. The trajectories in panels (a)-(d) and (f)-(h) are colored in the same way as the modulation path in (e). The octupole is depicted as a black circle with a paramagnetic director line of orientation $\omega$. We show the orientation of the octupole at six or three positions on each trajectory. A movie showing the dynamics of (a), (c), (d), (f) and (g) is provided with the Supplemental Material [32].

contain such irreversible jumps. For being able to adiabatically steer octupoles, it is important to know the number of minima per unit cell as well as the multiplicity of stable trajectories for a given modulation loop.

Figure 4 depicts the dynamic phase diagram of the topology of the position and orientation trajectories of a single octupole in the plane of tilt angle $\vartheta_{\text {ext }}$ and pattern phase $\varphi_{\mathrm{p}}$. Each point in the phase diagram characterizes the trajectory of an octupole driven by a modulation loop in which we adiabatically precess the external magnetic field at fixed tilt $\vartheta_{\text {ext }}$ around the $z$ axis.

The improper fourfold symmetry of the octupole is incompatible with the threefold symmetry of the pattern. Hence, in general there is no orientation of the external field allowing the octupoles to find a stable position near one of the threefold symmetric points $I_{1}, I_{2}$, and $I_{3}$. [There are only three particular orientations of $\mathbf{H}_{\mathrm{ext}}$ at the topological transition between two phases, where an octupole can reside above a threefold 

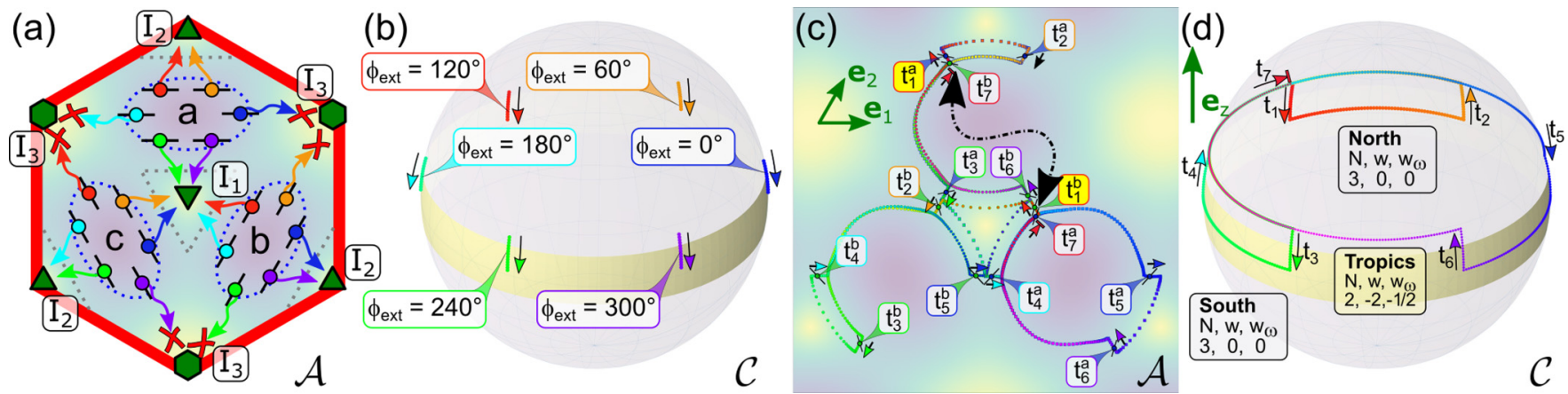

FIG. 5. Complex movements of octupoles in the action space $\mathcal{A}$ of a $C_{6}$ symmetric pattern $\left(\varphi_{\mathrm{p}}=0\right)$ driven by a complex modulation loop in control space $\mathcal{C}$. (a) Effective movement of the octupoles initially located close to one of the minima $a, b$, and $c$ to either $I_{1}$ or $I_{2}$ upon a transition of the driving field from the trivial phase in the north, $N, w, w_{\omega}=(3,0,0)$, to the nontrivial phase in the tropics, $N, w, w_{\omega}=(2,-2,-1 / 2)$ at different azimuths $\phi_{\text {ext }}$ as shown in (b). The colors of the octupoles in (a) and the transition angles in (b) match. Only two positions survive each transition as minima of the octupole potential. Minima moving towards $I_{3}$ (indicated by the red crosses) are annihilated. (c) Trajectories of two octupoles a (blue) and $\mathbf{b}$ (green) in $\mathcal{A}$ subject to the driving loop in $\mathcal{C}$ (d). The modulation switches between the trivial phase and the nontrivial phase and causes the exchange of the octupoles, i.e., the octupole a moves from the position marked as $t_{1}^{a}$ via $t_{i}^{a}, i \in\{2, \ldots, 7\}$ toward $t_{1}^{b}$ while the second octupole $\mathbf{b}$ moves from the position marked as $t_{1}^{b}$ via $t_{i}^{b}, i \in\{2, \ldots, 7\}$ toward $t_{1}^{a}$. The trajectories in (c) and the driving loop in (d) are colored in the same way. The unit vector $\mathbf{e}_{1}\left(\mathbf{e}_{2}\right)$ points along the direction of the lattice vector $\mathbf{a}_{1}\left(\mathbf{a}_{2}\right)$. A movie of the motion of both octupoles, a space-time plot together with the driving loop is provided with the Supplemental Material [32].

symmetric point, as visible in Fig. 4(d) where the adiabatic path crosses $I_{1}$.] From a topological point of view the space accessible to the octupoles is not simply connected. If $\varphi_{\mathrm{p}}=0$ the pattern has a proper sixfold symmetry at the point $I_{3}$.

Several characteristics of the octupole potential can be derived from symmetry arguments. For $\vartheta_{\text {ext }}=0$ (north pole of $\mathcal{C}$ ) the potential inherits the sixfold symmetry of the magnetic pattern and the octupole sits on a twofold symmetric point of the lattice which is commensurate with the particle symmetry. In each unit cell there are three minima called $a$, $b$, and $c$ [labeled in Fig. 5(a)] located at the twofold rotational symmetric points midway between the threefold symmetric points $I_{1}$ and $I_{2}$ of the lattice. Let $N$ be the number of coexisting octupole trajectories for a closed modulation loop. For $\varphi_{\mathrm{p}}=0$ and $\vartheta_{\mathrm{ext}} \approx 0, N=3$ equivalent octupole trajectories coexist (see Fig. 4). In each of the minima, $a, b$, and $c$, the diamagnetic axis is aligned along the $I_{1}-I_{2}$ direction [Fig. 4(f)]. These minima shift from these locations toward a new position slightly displaced towards the $I_{1}$ position if we change the pattern toward $\varphi_{\mathrm{p}}=\pi / 6$ for which the pattern acquires an improper sixfold symmetry at $I_{1}$. The improper sixfold symmetry causes the $I_{1}-I_{3}$ direction to be equivalent to the $I_{1}-I_{2}$ direction and for this reason there are now three additional minima between $I_{1}$ and $I_{3}$ where, in contrast to the other three minima the octupoles are oriented with their paramagnetic axis along the $I_{1}-I_{3}$ direction [Fig. 4(a)]. There is a transition in the amount of minima per unit cell from three minima $N=3$ [Figs. 4(b) and 4(f)] toward six minima $N=6$ [Fig. 4(a)] as we change $\varphi_{\mathrm{p}}$. Tilting the external field $\vartheta_{\text {ext }} \neq$ $0, \pi$ breaks the threefold symmetry and this eventually reduces the number of minima per unit cell [see, e.g., Figs. 4(c) and $4(\mathrm{~g})]$.

Consider a sixfold pattern with $\varphi_{\mathrm{p}}=0$. If we move away from the north pole of $\mathcal{C}$ to the equator, say $\mathbf{H}_{\text {ext }}^{1}$, we find at least one minimum of the potential in $\mathcal{A}$ that is not the twofold symmetric point. The point $-\mathbf{H}_{\mathrm{ext}}^{1}$ (opposite to $\mathbf{H}_{\mathrm{ext}}^{1}$ on $\mathcal{C}$ ) is a mirror image of $\mathbf{H}_{\mathrm{ext}}^{1}$. Thus, a point mirror symmetric to the twofold symmetric point in $\mathcal{A}$ must be a minimum for the mirror symmetric orientation $-\mathbf{H}_{\mathrm{ext}}^{1}$. However, if we reverse the magnetic field, all minimum positions of $U_{\text {oct }}$ remain the same due to the symmetry of an octupole, but the octupole orientation rotates by $\omega \rightarrow \omega \pm \pi / 2$. For this reason, both minima (the original one and the one for the reversed field) must be minima to both field orientations $\mathbf{H}_{\mathrm{ext}}^{1}$ and $-\mathbf{H}_{\mathrm{ext}}^{1}$. Hence, if $\varphi_{\mathrm{p}}=0$ and $\vartheta_{\mathrm{ext}}=\pi / 2$ the minima of $U_{\mathrm{oct}}$ occur in pairs lying symmetric on opposite sides of the $I_{1}, I_{2}$ midpoints. The two octupoles residing in such pair of independent minima have a relative orientation of $\Delta \omega=\pi / 2$. For $\varphi_{\mathrm{p}}=0$ and $\vartheta_{\mathrm{ext}}=\pi / 2$ we find only two trajectories, $N=2$, per unit cell that coexist over a driving loop [see Fig. 4(h)]. The twofold symmetry of the midpoints between $I_{1}$ and $I_{2}$ is broken if $\varphi_{\mathrm{p}} \neq 0$ such that for larger $\varphi_{\mathrm{p}}$ and $\vartheta_{\mathrm{ext}} \approx \pi / 2$ only one trajectory per unit cell exists over a driving loop, $N=1$ [see Fig. 4(d)].

\section{B. Topology of the trajectories}

Next, we topologically characterize the adiabatic trajectories of the octupoles. Recall that we loop the magnetic field at constant tilt $\vartheta_{\text {ext }}$ around the $z$ axis $\left(\phi_{\text {ext }} \rightarrow \phi_{\text {ext }}+2 \pi\right)$. We define three integer winding numbers $w_{1}, w_{2}$, and $w_{3}$ of the trajectories around the threefold points $I_{1}, I_{2}$, and $I_{3}$ in action space $\mathcal{A}$, respectively. The winding numbers count the turns of a particle on a trajectory around these points. All trajectories are related by symmetry and we can unambiguously characterize a dynamical phase by the total winding number $w=w_{1}+w_{2}+w_{3}$ since at most one $w_{i} \neq 0$. Additionally, we define the half integer orientational winding number $w_{\omega}=\frac{1}{2 \pi} \Delta \omega$ that counts the total rotation $\Delta \omega$ of the octupole along its own $z$ axis during one closed loop. Positive (negative) values of all winding numbers correspond to the same (opposite) sense of rotation as the driving field.

For small tilt angles either three or six trajectories coexist [Figs. 4(a), 4(b), and 4(f)]. All trajectories are topologically trivial since all, positional and orientational, winding 
numbers vanish. At larger tilt angle we find either one or two trajectories. For close to sixfold symmetric patterns the trajectories appear in pairs of two, one nontrivially winding around $I_{1}\left(w_{1}=-2, w_{\omega}=-1 / 2\right)$, the other nontrivially winding around $I_{2}\left(w_{2}=-2, w_{\omega}=-1 / 2\right)$ [see Figs. 4(g) and 4(h)]. The nontrivial loops are also nontrivial with respect to the orientation. The transitions between topologically trivial $(w=0)$ and nontrivial $(w \neq 0)$ phases occur via intervening nonadiabatic ratchets where the octupoles irreversibly jump from one location to another at a particular $\phi_{\text {ext }}$ of the loop. When we increase $\varphi_{\mathrm{p}}$ only one nontrivial trajectory $\left(w_{1}=-2, w_{\omega}=\right.$ $-1 / 2$ ) remains stable and the other trajectory around $I_{2}$ is no longer stable [see Fig. 4(d)]. If we decrease the tilt angle and move toward $\varphi_{\mathrm{p}}=\pi / 6$ we eventually cross a topological transition line toward one single trajectory with winding numbers $w_{1}=1, w_{\omega}=-1 / 2$ [see Fig. 4(c)]. The winding numbers not explicitly mentioned vanish.

\section{Braiding with octupoles}

The existence of dynamical phases in which several octupole trajectories coexist allows the independent and simultaneous steering of identical octupoles. That is, using a single external field we can independently control the motion of identical particles. To illustrate this we use complex driving loops of the external field on a sixfold symmetric pattern to braid and weave with octupoles.

The octupoles are placed initially in nonequivalent minima of $U_{\text {oct }}$. The driving loops consist of (precession) segments of any $\Delta \phi_{\text {ext }}$ in either of both phases $N, w, w_{\omega}=(3,0,0)$ and $N, w, w_{\omega}=(2,-2,-1 / 2)$. These segments are connected by straight crossings of the topological transition between those phases at specific $\phi_{\text {ext }}$. The succession of crossings determines the steering of the octupoles. In Fig. 5(a) we show the effect of the $N=3 \rightarrow N=2$ transition at different $\phi_{\text {ext }}$ on the three initially possible nonequivalent octupoles of one unit cell. As only two of them survive the transition, we have to make sure only these two nonequivalent positions are filled. The nonsurviving octupole will relax irreversibly into one of the other minima of $U_{\text {oct }}$. The opposite transition is not harmful, as it creates an empty, unoccupied minimum.

Arranging commands in the right order allows one to move octupoles in any desired way through the lattice. We can, e.g., exchange two octupoles on a proper sixfold pattern. In Fig. 5(d) we show a driving loop that switches back and forth between the phases $N, w, w_{\omega}=(3,0,0)$ and $N, w, w_{\omega}=$ $(2,-2,-1 / 2)$ three times and causes the exchange of two octupoles residing in two distinct minima of $U_{\text {oct }}$ [see Fig. 5(c)]. We also show the two trajectories that guide the two octupoles from their start location toward the start location of the other particle.

Highly complex motion such as braiding and weaving with octupoles can be programed. For braiding with three octupoles we place two octupoles in neighboring equivalent minima and the third one in a nonequivalent minimum. The modulation loop then successively winds the nonequivalent octupole around one of the two equivalent particles. Figure 6(a) shows a space-time plot of the three trajectories that we have deformed into a standard braid, without changing the topology of the space-time trajectories.
It is possible to weave a space-time carpet by placing octupoles on a line of alternating minima and then successively half-winding the nonequivalent octupole around the set of equivalent octupoles. The trajectory of the nonequivalent octupole is an intercellular trajectory, while the equivalent octupoles remain close to their initial positions. In Fig. 6(b) we show a minimalist example of weaving together with the generating modulation loop.

\section{CONCLUSIONS}

We propose to use the complex topology of the potential of colloidal octupoles above a periodic lattice for the parallel and simultaneous steering of identical particles into different directions driven by a single homogeneous magnetic field. This allows us to create braids and carpets of any structure, as the control loop can be adapted throughout the procedure and is not restricted to a predefined structure as in, e.g., Ref. [26]. In electronic quantum states the semiclassical topology of Bloch states remains preserved if tunneling between different bands is prevented by a large enough band gap. Applying a homogeneous external field to independently but simultaneously control the motion of equivalent quantum particles (instead of colloidal particles) on nonequivalent positions of a pattern should be feasible as well as long as the barrier between different minima is high enough to prevent tunnel processes. Such quantum octupoles could be achieved by miniaturization of the entire setup, the pattern, and the octupoles, to the nanoscale. Our control loops (specified by, e.g., the series of transition times in Fig. 6) would provide an explicit protocol to braid with anyonic quantum octupoles, which is a current problem of quantum computation $[11,33]$. Regarding classical applications, colloidal particles can be used as drug carriers on lab-on-a-chip devices. With our methodology it could be possible to deliver payloads to distinct sites in a particular order using just one global external control field and colloidal octupoles.

We have demonstrated how to cause the exchange of two particles using a global spatially homogeneous field to address two similar particles in two different ways. A technical method that externally achieves such exchange in miniaturized quantum particle systems is still missing. The topological robustness of our process might survive a miniaturization to the quantum scale and would thus indeed be quite a useful method.

\section{ACKNOWLEDGMENTS}

T.L. acknowledges financial support by the program "Biological Physics" of the Elite Network of Bavaria. We acknowledge funding by the Deutsche Forschungsgemeinschaft (DFG, German Research Foundation) under Project No. 440764520.

\section{APPENDIX: BROWNIAN DYNAMICS SIMULATIONS}

The trajectories are calculated using Brownian dynamics simulations. Time discretization of the overdamped equation of motion of the colloidal octupoles using the standard Euler 


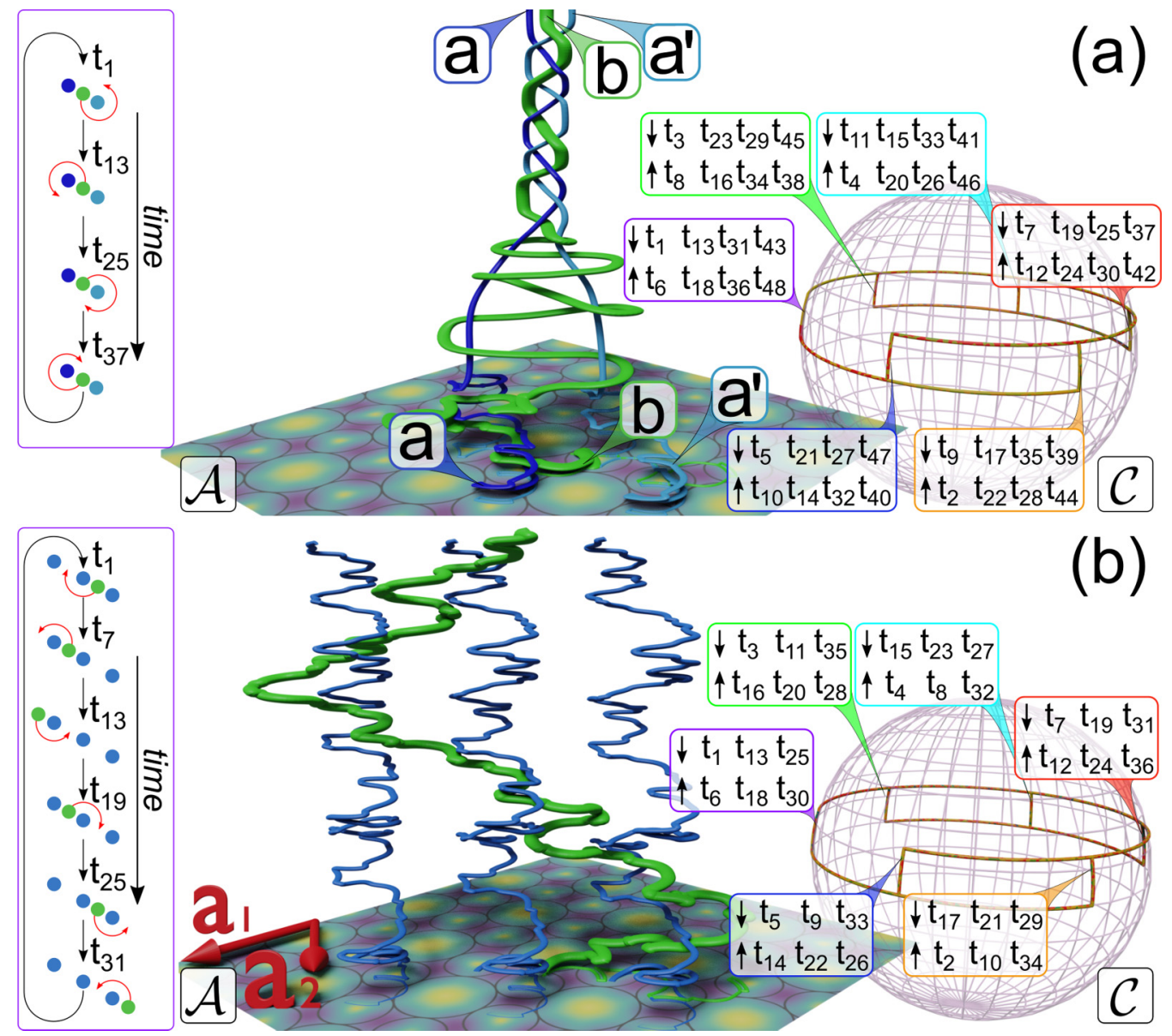

FIG. 6. (a) Trajectories of three octupoles forming a braid using two octupoles a and $\mathbf{a}^{\prime}$ (blue) initially placed in equivalent potential minima of two different unit cells and a third octupole placed in a nonequivalent minimum $\mathbf{b}$ (green). Virtual strings colored according to the corresponding octupoles indicate the movement of the octupoles in time. The braid can be seen at the top of the image, where we have deformed the trajectories into the standard braid representation without altering the topology. (b) Space-time plot of the trajectories of a series of equivalent octupoles (blue) woven together by a fourth octupole (green) following a nonequivalent minimum position of the octupole potential. The complex modulation loop in control space $\mathcal{C}$ used to drive the motion is shown on the right via the order of the transition times $t_{i}$, $i \in\{1, \ldots, 36\}$ between the $N, w, w_{\omega}=(3,0,0)$ phase and the $N, w, w_{\omega}=(2,-2,-1 / 2)$ phase. The paths in action space $\mathcal{A}$ are sketched by vanishing lines on top of the $C_{6}$ symmetric pattern. $\mathbf{a}_{1}$ and $\mathbf{a}_{2}$ are the lattice vectors of the pattern. The sketches on the left show the schematic exchange or winding of the particles. Movies of the motion of the octupoles, a space-time plot, and the driving loop are provided with the Supplemental Material [32].

algorithm results in

$$
\mathbf{r}_{i}(t+\Delta t)=\mathbf{r}_{i}(t)-\frac{\Delta t}{\gamma} \nabla U_{\mathrm{oct}}\left(\mathbf{r}_{i}, \omega_{i}, \mathbf{H}_{\mathrm{ext}}, t\right)+\eta_{i}(t)
$$

where $\mathbf{r}_{i}$ is the position in action space $\mathcal{A}$ of the $i$ th octupole, $\Delta t$ is the time step of the simulation, $\gamma$ is the friction coefficient against the (implicit) solvent, $\omega_{i}$ is the orientation of the $i$ th octupole (angle between its paramagnetic axis and the lattice primitive vector $\mathbf{a}_{1}$ ), and $\boldsymbol{\eta}_{i}$ is a delta-correlated Gaussian random displacement with standard deviation $\sqrt{2 \Delta t k_{B} T / \gamma}$. Here $k_{B}$ is the Boltzmann constant and $T$ is absolute temperature.

We work in units of the magnitude of the first lattice vector $a_{1}$, the friction coefficient $\gamma$, and the magnitude of the induced magnetic octupole moment $q_{\text {oct }} H_{\text {ext }}$ times the saturation magnetization of the pattern $M_{\mathrm{p}}$ that is $q_{\mathrm{oct}} H_{\mathrm{ext}} M_{\mathrm{p}}$. Hence, the time is given in units of $\tau=\gamma a_{1}^{4} /\left(q_{\mathrm{oct}} H_{\mathrm{ext}} H_{\mathrm{p}}\right)$, and the energy in units of $\epsilon=q_{\mathrm{oct}} H_{\mathrm{ext}} H_{\mathrm{p}} / a_{1}^{2}$. The time step of the simulation is $d t / \tau=1 / 450000$. The period of one loop of the external field is $T_{0} / \tau \approx 1$ and the temperature is set to $k_{B} T / \epsilon=10^{-3}$ since we are only interested in the adiabatic regime for which the Brownian motion does not play any role. Nevertheless, due to the topological character of the motion, the trajectories are robust against thermal perturbation as we have demonstrated in Ref. [6] for the case of dipolar particles.

We neglect interparticle interactions between colloidal octupoles, which is not problematic since the particles stay always far away from each other due to the external fields and the reduced size of the particles as compared to the size of the unit cell. Note that the octupole-octupole interaction decays very fast with the interparticle distance. To speed up the simulation, we calculate at each time step the orientations $\omega_{i}$ using the Hessian matrix of the dipole potential, i.e.,

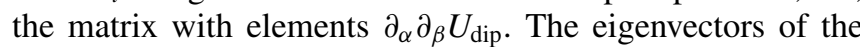
Hessian matrix give the orientation of the paramagnetic and diamagnetic axes and hence the orientations $\omega_{i}$. We therefore 
consider that the orientational relaxation of the octupoles is fast compared to the rotational diffusion, which constitutes a very good approximation in the adiabatic limit. The inclusion of free diffusion of particle orientations might play a role in ratchet events and under strong driving conditions that might drive the system out of the adiabatic regime.
[1] L. Gao, M. A. Tahir, L. N. Virgin, and B. B. Yellen, Multiplexing superparamagnetic beads driven by multi-frequency ratchets, Lab Chip 11, 4214 (2011).

[2] K. Kataoka, A. Harada, and Y. Nagasaki, Block copolymer micelles for drug delivery: Design, characterization and biological significance, Adv. Drug Deliv. Rev. 47, 113 (2001).

[3] H. Löwen, Colloidal dispersions in external fields: Recent developments, J. Phys.: Condens. Matter 20, 404201 (2008).

[4] A. Erbe, M. Zientara, L. Baraban, C. Kreidler, and P. Leiderer, Various driving mechanisms for generating motion of colloidal particles, J. Phys.: Condens. Matter 20, 404215 (2008).

[5] J. Loehr, D. de las Heras, M. Lönne, J. Bugase, A. Jarosz, M. Urbaniak, F. Stobiecki, A. Tomita, R. Huhnstock, I. Koch, A. Ehresmann, D. Holzinger, and T. M. Fischer, Lattice symmetries and the topologically protected transport of colloidal particles, Soft Matter 13, 5044 (2017).

[6] D. de las Heras, J. Loehr, M. Lönne, and T. M. Fischer, Topologically protected colloidal transport above a square magnetic lattice, New J. Phys. 18, 105009 (2016).

[7] J. Loehr, D. de las Heras, A. Jarosz, M. Urbaniak, F. Stobiecki, A. Tomita, R. Huhnstock, I. Koch, A. Ehresmann, D. Holzinger, and T. M. Fischer, Colloidal topological insulators, Commun. Phys. 1, 4 (2018).

[8] H. Massana-Cid, A. Ernst, D. de las Heras, A. Jarosz, M. Urbaniak, F. Stobiecki, A. Tomita, R. Huhnstock, I. Koch, A. Ehresmann, D. Holzinger, and T. M. Fischer, Edge transport at the boundary between topologically equivalent lattices, Soft Matter 15, 1539 (2019).

[9] M. Mirzaee-Kakhki, A. Ernst, D. de las Heras, M. Urbaniak, F. Stobiecki, J. Gördes, M. Reginka, A. Ehresmann, and T. M. Fischer, Simultaneous polydirectional transport of colloidal bipeds, Nat. Commun. 11, 4670 (2020).

[10] J. Loehr, M. Lönne, A. Ernst, D. de las Heras, and T. M. Fischer, Topological protection of multiparticle dissipative transport, Nat. Commun. 7, 11745 (2016).

[11] C. Nayak, S. H. Simon, A. Stern, M. Freedman, and S. Das Sarma, Non-Abelian anyons and topological quantum computation, Rev. Mod. Phys. 80, 1083 (2008).

[12] F. D. M. Haldane, Fractional Statistics in Arbitrary Dimensions: A Generalization of the Pauli Principle, Phys. Rev. Lett. 67, 937 (1991).

[13] W. Ling, J. N. Chung, T. R. Troutt, and C. T. Crowe, Direct numerical simulation of a three-dimensional temporal mixing layer with particle dispersion, J. Fluid Mech. 358, 61 (1998).

[14] J. M. Wheaton, J. Brasington, S. E. Darby, A. Kasprak, D. Sear, and D. Vericat, Morphodynamic signatures of braiding mechanisms as expressed through change in sediment storage in a gravel-bed river, J. Geophys. Res: Earth. Sci 118, 759 (2013).

[15] G.-P. Yang, L. Hou, X.-J. Luan, B. Wu, and Y.-Y. Wang, Molecular braids in metal-organic frameworks, Chem. Soc. Rev. 41, 6992 (2012).

[16] X.-J. Luan, X.-H. Cai, Y.-Y. Wang, D.-S. Li, C.-J. Wang, P. Liu, H.-M. Hu, Q.-Z. Shi, and S.-M. Peng, An investigation of the self-assembly of neutral, interlaced, triple-stranded molecular braids, Chem. Eur. J. 12, 6281 (2006).

[17] D.-R. Xiao, Y.-G. Li, E.-B. Wang, L.-L. Fan, H.-Y. An, Z.-M. $\mathrm{Su}$, and $\mathrm{L}$. $\mathrm{Xu}$, Exceptional self-penetrating networks containing unprecedented quintuple-stranded molecular braid, 9-fold meso helices, and 17-fold interwoven helices, Inorg. Chem. 46, 4158 (2007).

[18] X.-J. Luan, Y.-Y. Wang, D.-S. Li, P. Liu, H.-M. Hu, Q.-Z. Shi, and S.-M. Peng, Self-assembly of an interlaced triplestranded molecular braid with an unprecedented topology through hydrogen-bonding interactions, Angew. Chem., Int. Ed. 44, 3864 (2005).

[19] S. Copar, U. Tkalec, I. Muševič, and S. Žumer, Knot theory realizations in nematic colloids, Proc. Natl. Acad. Sci. USA 112, 1675 (2015).

[20] M. Ravnik and and S. Žumer, Nematic colloids entangled by topological defects, Soft Matter 5, 269 (2009).

[21] M. R. Dennis, R. P. King, B. Jack, K. O'Holleran, and M. J. Padgett, Isolated optical vortex knots, Nat. Phys. 6, 118 (2010).

[22] C. Moore, Braids in Classical Dynamics, Phys. Rev. Lett. 70, 3675 (1993).

[23] M. S. Alber and J. E. Marsden, On geometric phases for soliton equations, Commun. Math. Phys. 149, 217 (1992).

[24] P. Pieranski, S. Clausen, G. Helgesen, and A. T. Skjeltorp, Braids Plaited by Magnetic Holes, Phys. Rev. Lett. 77, 1620 (1996).

[25] J.-B. Caussin and D. Bartolo, Braiding a Flock: Winding Statistics of Interacting Flying Spins, Phys. Rev. Lett. 114, 258101 (2015).

[26] C. P. Goodrich and M. P. Brenner, Using active colloids as machines to weave and braid on the micrometer scale, Proc. Natl. Acad. Sci. USA 114, 257 (2017).

[27] M. J. Bowick and L. Giomi, Two-dimensional matter: Order, curvature and defects, Adv. Phys. 58, 449 (2009).

[28] A. Ray and T. M. Fischer, Magnetic field controlled composite paramagnetic-diamagnetic colloidal phases, J. Phys. Chem. B 116, 8233 (2012).

[29] K. S. Khalil, A. Sagastegui, Y. Li, M. A. Tahir, J. E. S. Socolar, B. J. Wiley, and B. B. Yellen, Binary colloidal structures assembled through Ising interactions, Nat. Commun. 3, 794 (2012).

[30] C. Chappert, H. Bernas, J. Ferré, V. Kottler, J.-P. Jamet, Y. Chen, E. Cambril, T. De-Volder, F. Rousseaux, V. Mathet, and H. Launois, Planar patterned magnetic media obtained by ion irradiation, Science 280, 1919 (1998).

[31] P. Kuświk, A. Ehresmann, M. Tekielak, B. Szymański, I. Sveklo, P. Mazalski, D. Engel, J. Kisielewski, D. Lengemann, M. Urbaniak, C. Schmidt, A. Maziewski, and F. Stobiecki, Colloidal domain lithography for regularly arranged artificial magnetic out-of-plane monodomains in $\mathrm{Au} / \mathrm{Co} / \mathrm{Au}$ layers, Nanotechnology 22, 095302 (2011).

[32] See Supplemental Material at http://link.aps.org/supplemental/ 10.1103/PhysRevResearch.3.013043 for videos of the trajectories followed by the colloidal octupoles.

[33] A. Kitaev, Anyons in an exactly solved model and beyond, Ann. Phys. (NY) 321, 2 (2006). 